\title{
Studies of Transgenic Mosquitoes in Disease-Endemic Countries: Preparation of Containment Facilities
}

\author{
M. Megan Quinlan,, James Mutuku Mutunga, ${ }^{2,3, *}$ Abdoulaye Diabaté, ${ }^{4}$ Moussa Namountougou, \\ Mamadou B. Coulibaly, Lakamy Sylla, Jonathan Kayondo, Victor Balyesima,, Lorna Clark, \\ Mark Q. Benedict, and Peter Raymond ${ }^{9}$
}

\begin{abstract}
Novel approaches to area-wide control of vector species offer promise as additional tools in the fight against vectored diseases. Evaluation of transgenic insect strains aimed at field population control in disease-endemic countries may involve international partnerships and should be done in a stepwise approach, starting with studies in containment facilities. The preparations of both new-build and renovated facilities are described, including working with local and national regulations regarding land use, construction, and biosafety requirements, as well as international guidance to fill any gaps in regulation. The examples given are for containment categorization at Arthropod Containment Level 2 for initial facility design, classification of wastes, and precautions during shipping. Specific lessons were derived from preparations to evaluate transgenic (non-gene drive) mosquitoes in West and East African countries. Documented procedures and the use of a non-transgenic training strain for trial shipments and culturing were used to develop competence and confidence among the African facility staff, and along the chain of custody for transport. This practical description is offered to support other research consortia or institutions preparing containment facilities and operating procedures in conditions where research on transgenic insects is at an early stage.
\end{abstract}

Keywords: biosafety, compliance, containment, insectary, mosquitoes, transgenic

\section{Introduction}

C ONTROL OF MANY INSECT VECTOR or pest populations can be achieved through release of mass-reared sterile insects of the same species in an area-wide approach (e.g., Klassen and Curtis 2005). There is increasing interest in the use of heritable traits in mass-reared insect releases to increase efficiency and reduce costs (Burt 2014). When these insects, or any organisms, incorporate new traits or are produced with novel methods or approaches, a stepwise approach is recommended to establish safety and efficacy through studies in laboratory conditions and limited-area releases, before openfield applications (Benedict et al. 2008, Marris and Jefferson 2013, WHO/TDR and FNIH 2014, WHO/TDR 2015). This is especially true when novel traits will persist or be spread through the existing insect population (NASEM 2016).

Presently, much of the development of transgenic insect strains has occurred outside the geographic areas targeted for

\footnotetext{
${ }^{1}$ Centre for Environmental Policy, Imperial College London, Ascot, United Kingdom.

${ }^{2}$ International Centre of Insect Physiology and Ecology, Mbita Point, Kenya.

${ }^{3}$ Department of Biological Sciences, Mount Kenya University, Thika, Kenya.

${ }^{4}$ Institut de Recherche en Sciences de la Santé, (IRSS)/Centre Muraz, Bobo Dioulasso, Burkina Faso.

${ }^{5}$ Malaria Research and Training Center, Université des Sciences, des Techniques et des Technologies de Bamako (MRTC/USTTB), Bamako, Mali.

${ }^{6}$ Uganda Virus Research Institute (UVRI), Entebbe, Uganda.

${ }^{7}$ Department of Life Sciences, Imperial College London, Ascot, United Kingdom.

${ }^{8}$ Entomology Branch, Centers for Disease Control and Prevention (CDC), Atlanta, Georgia.

${ }^{9}$ Donald Danforth Plant Science Center, St. Louis, Missouri.

*Current affiliation: Department of Biological Sciences, Mount Kenya University, Thika, Kenya.
}

(C) M. Megan Quinlan et al. 2018; Published by Mary Ann Liebert, Inc. This is an Open Access article distributed under the terms of the Creative Commons Attribution License, which permits unrestricted use, distribution, and reproduction in any medium, provided the original work is properly cited. 
their use, with further research and development occurring within the disease-endemic countries (DEC). Research partners in the countries targeted for ultimate use of the genetic strategies provide international consortia not only with local expertise and technical experience, but also with an institutional host for both contained studies and eventual confined field studies and, critically for regulatory submissions, a legal entity in country.

Such studies are designed to answer questions from researchers, regulators, and other stakeholders and to form a basis of future applications for confined field studies. Preparations comprise facility design and construction or refurbishment, official recognition or approval of the containment facility, appropriate permitting of the activities to take place in the facility, and preparation of staff to manage and operate the containment facility for colony maintenance and studies. Although finding research partners is not discussed in this study, it requires an advanced assessment of key needs and the availability of experienced, suitable staff.

Under the scenario described in this article, partner institutions in the countries affected by the targeted disease need to be prepared for receiving transgenic insects from abroad and for their use in either contained studies or, after augmentation of colonies, for larger scale experiments in local field conditions. The import of transgenic strains manifests as further requirements than if the transgenic strain had been developed in country. (Use of other forms of modified insects may reveal the lack of clear regulatory pathways). This article presents general recommendations from the perspective of an institution in a DEC partner country that must work with a combination of the national regulatory context and by following relevant international guidance. Finally, more specific experiences of a single project preparing for studies on transgenic mosquitoes are presented. The article does not address safety considerations for workers related to bacteria, viruses, or other parasites, but rather concentrates on the prevention of accidental release of transgenic insects into the environment during the study phase or when augmenting production for larger trials.

Selection of a site for field studies on novel mosquito technologies has been discussed elsewhere (Lavery et al. 2008, Marsden et al. 2013, Brown et al. 2014, Iyaloo et al. 2014). Choosing a site for a research or production facility is subject to other types of laws and regulations (IAEA 2008). Figure 1 (in a style similar to Mourya et al. 2014) illustrates some of the steps toward establishing a new containment facility appropriate for transgenic insects, with details for the case of mosquitoes (flying stage and blood fed). Additional steps for working with strains containing a gene drive mechanism would include review of the land in terms of potential for an ecological buffer zone outside the facility (step 2) and some additional specifications (step 9) such as controlled directional air flow in the entry vestibule, in addition to double locking doors (Benedict et al. 2018).

\section{Environmental impact, building permits, and land use zoning}

As with any other building project, the client must secure a planning permit, normally from the subregional (state,
2. Review of land ownership, zoning, permits

3. Proposal for funding or finalization of budget

4. Formulation of institutional or project committee to oversee development process

5. Final design and request for bids

6. Cooperation agreement with external funder, laying out any expectations, ownership, etc.

7. Hiring of contractor for construction

8. Detailed drawings for final discussions

9. Specifications for containment related aspects

10. Environmental Impact Assessment if required

11. Monitoring of construction including added containment measures

12. Choosing right materials for detecting free fliers and to withstand humidity

13. Setup of insectary environmental monitors, sources of humidity and temperature control

14. Installation of filters, screens, and autoclave, verification of containment measures

15. Verification of readiness of final stage of waste stream (e.g. incinerator, septic tank) in terms of planned containment measures; readiness of animal house if required for blood

16. Installation and testing of minor equipment, including controlled access equipment (swipe entry, keypad, surveillance camera or other methods)

17. Portable Appliance Testing and/or setup of surge protection and electricity backup devices

18. Filing of owner's manuals and installation certificates for all equipment

19. Development of a maintenance plan or service agreement
1. Construction site selection

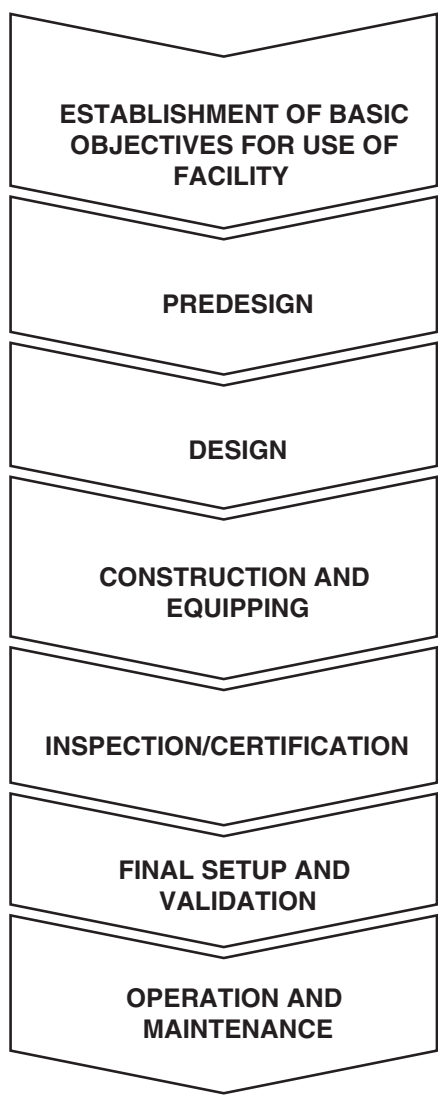

FIG. 1. Summary of the construction and equipping steps performed in preparation of an arthropod containment facility for studies of transgenic mosquitoes in example disease endemic countries. 
province, county, or district) and/or local (municipal) government office before commencing the construction process. The DEC partner institute may well be a public entity itself, and procedures for government projects may come into play. Unless the facility is situated on a government or university campus, the surrounding neighborhood and any likely changes over subsequent years will need to be considered to determine if the facility is appropriate to the assigned zoning of the site. Many countries may have a nascent system for zoning or land use planning in urban areas, but it is worth remembering that a containment facility for such small-scale studies is unlikely to produce any hazardous or biomedical waste, cause a high noise level, and alter road use and volume of traffic, or any of the other factors that might shift a site toward a higher industrial or medical category. Environmental regulations may influence siting of an animal house, which could be needed for extracting blood for vector feeding, or for composting of insect diet if large quantities of treated waste are to be managed. Backup generators are needed for any facility of this nature. Common sense location of an incinerator, needed to complete the waste stream special handling, would avoid smoke going toward other work or inhabited areas.

If a detailed Environmental Impact Assessment (EIA) or similar study for the facility is required, this generally must be submitted through an environmental engineer qualified and certified in the country. For example, a containment facility built on the Mbita Point campus of the International Centre of Insect Physiology and Ecology (ICIPE) in Kenya was subject to an EIA during the planning stage. The contracted consultant submitted an EIA report to the National Environmental Management Authority (NEMA), which is the relevant regulatory agency in Kenya established under the Environmental Management and Coordination Act No. 8 of 1999 (Kenya 1999). Upon approval by NEMA, the regular process for issuing a building permit took place.

In general, however, a structure for the purpose of small- to medium-scale transgenic research is not likely to be large enough to require an EIA in many cases, especially if situated within a government or university campus. Obtaining a building permit does not preclude the need for a study permit. If the scope of the EIA is not limited to the potential impact of the facility-build and extends to issues around the studies to be undertaken, which is under the mandate of the national biosafety authority (NBA) when using transgenic insects, requirements and monitoring may become duplicative and will require a particular expertise.

\section{Containment Facility Design, Creation, and Approval}

\section{Designing and overseeing a new build}

The design of a purpose-made building for contained studies of transgenic insects must meet any containment feature stipulated in national containment guidelines at the appropriate classification level (see Determination of the required containment level). The researchers should address the intentions of the regulations, possibly adding features according to the study organism, such as features related to risks from flying insects (Quinlan 2014) or enhanced management for strains containing gene drive mechanisms. If containment and/or biosafety regulations or official guidance do not exist in the country, the researchers and ap- propriate governmental authority may wish to reference internationally recognized guidance such as the Arthropod Containment Guidelines, a consensus document developed by the American Committee of Medical Entomologists, which has since been applied globally (ACME/ASTMH 2003).

To ensure that the building contractors are qualified, the specification of work may state that some specialized skills beyond the usual construction experience are required. Paramount to success is the ability to take instruction and collaborate with researchers, while possibly educating and offering counterproposals to meet their objectives. Therefore, the tendering process must take into account experience with laboratory construction and quality control measures, and not only costs. Poor or inappropriate workmanship may result in the building being deemed not fit for purpose and unusable for the research.

Likewise, the selection of an architect and engineers to oversee the construction should be done through a rigorous competitive process. Given the demands of the build, there might be a specialist role for someone able to understand and "translate"' what scientists need to the engineers, although an architect with these skills could potentially play that role. It is critical that whoever takes on the role is independent from engineers and companies involved in the project. Any subcontractor subsequently sourced by the contractor or the partner institute ("client") must be vetted and approved by the architect and engineers. All work by the contractor or subcontractors must be officially assigned by the architect. All firms involved in the construction must be registered with relevant national government agencies and be licensed to operate. Payments to suppliers and service providers are made only after the architect issues a payment note (on behalf of all the contractors) and these must comply with applicable country taxation laws.

When architects and engineers are selected, the draft floor plans and architectural rendering of the containment facility should be developed in collaboration between this team and the scientists (entomologists and molecular biologists) conversant with the appropriate containment measures, national biosafety laws, and the work to be performed in the facility. For example, the widely adopted Arthropod Containment Level 2 (ACL-2) (ACME/ASTMH 2003) requires controlled access through a vestibule with a system of double locking doors, which cannot be opened simultaneously. For higher risk research (e.g., strains with gene drive), a negative pressure zone or other features may be needed in the main entry vestibule, or even between outer and inner rooms providing additional layers of containment. All consultations relating to the build-design and actual building of the facility must involve engagement with the researchers who will be using it, either through a designated coordinator/build manager or the Principal Investigator (PI) funding the build.

The floor plan and internal flow of foot traffic should suit the planned use of the facility. Arthropod rearing rooms, commonly referred to as the insectary (although sometimes the entire facility is referred to this way), should provide adequate space and their location/layout should allow movement between the rooms (e.g., for polymerase chain reaction, microscopy, and autoclave), while minimizing unnecessary entry into other laboratory areas or offices. The space needed for the required output of mosquitoes should 
be calculated to allow for the most appropriate configuration for work flow (e.g., Balestrino et al. 2012). Doors that are well sealed when closed to prevent escape of insects will provide the interconnection between the insectary and these other rooms. The design must anticipate whether the entire building or only certain rooms will be designated as areas for contained use permit activities, as this would alter the design of such interconnections considerably. Laboratory space should be planned to allow convenient transfer of research material, equipment, and consumables into the facility without passing through the insectary areas, while movement of the insects themselves should be minimized. Furthermore, the siting/ design of culture/insectary rooms, office space, laboratory space, stores, and other rooms must be determined early in the building design since their locations affect other downstream support entities such as plumbing, waste disposal, data cabling, and installation of environmental control units.

A set of professional drawings of the floor plans with each of the incorporated containment measures will be useful at the time of application to the NBA for certification. Figure 2 shows a generic floor plan of a containment facility featuring insectaries, in this case for transgenic mosquitoes, with functions of each room labeled. Although layout may vary significantly according to the organism under study, this provides an example of a small facility designed to allow the work flow needed for insect colony maintenance and studies.

During the construction phase, all material brought on-site should undergo relevant structural test analysis and reports must be verified by the architect and engineers. There should be routine on-site inspection visits (e.g., every 2 weeks) to evaluate progress, inspect the quality of work, and generate timelines for the next phase of work, aligned to a comprehensive completion program. The construction inspection visits should be coordinated by the architect and engineers, and attended by scientists (users), the client (institute) estate staff, station managers, contractors, and subcontractors. Strict adherence to the completion schedule is monitored during the routine visits. A checklist of completed/pending items should also be circulated during each visit as well as notes on issues that are pertinent to the parties involved.
Thought should be given to the equipping of the facility at the early planning stages to allow for large items of equipment to be placed in practical locations. Heavy bench-top centrifuges may require additional support and autoclaves may need specialist installation with venting and special water supplies to allow for steam outlets, and in some instances, a three-phase power supply. In some cases, a separate on/off switch and/or transformer, if equipment is procured internationally, in an easily accessible location may need to be added, to ensure the safety of operating equipment. Electric outlets also may be needed at ceiling level for installing fans, insect traps, fire alarm systems, or environmental monitors.

A maintenance program for all essential items should be put into place to ensure that the equipment is regularly serviced. If working across multiple facilities, it may be advisable to procure any essential, large items of equipment from a single source, although equipment procured from Europe, for example, may have different specifications. This leads to standardization of methods across sites and may give some leverage for cost savings (although the time and cost of shipping and clearance through customs must be taken into account). However, it is important that common consumables be procured locally for ease of laboratory management. If consumables and supplies are to be procured overseas, a stock management system that takes into account proper planning for overseas orders must be developed and adhered to. Frequent changes in suppliers of some materials, such as for insect diet or cleaning products, including bleach, may result in variation of quality and affect the health of the colonies (a key influence on the usefulness of research data, as discussed in Quinlan et al. 2018).

Details of the decor should be suited to the need to easily view the surfaces of walls and ceilings, to ensure adult mosquitoes outside rearing cages can be detected and killed. The choice of paint, counter tops, cupboard finishes, and furnishing should anticipate the potential for volatile compounds that might affect mosquito rearing, as well as the impact over time of high humidity in warm environments required for insectaries. New decor may require a resting period to allow toxic fumes to dissipate, before introducing colonies to the facility.
A

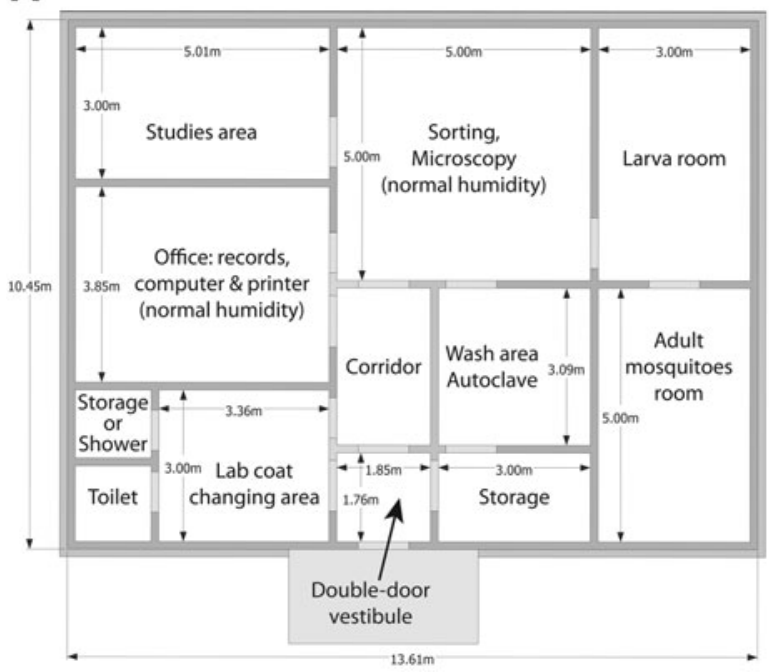

B

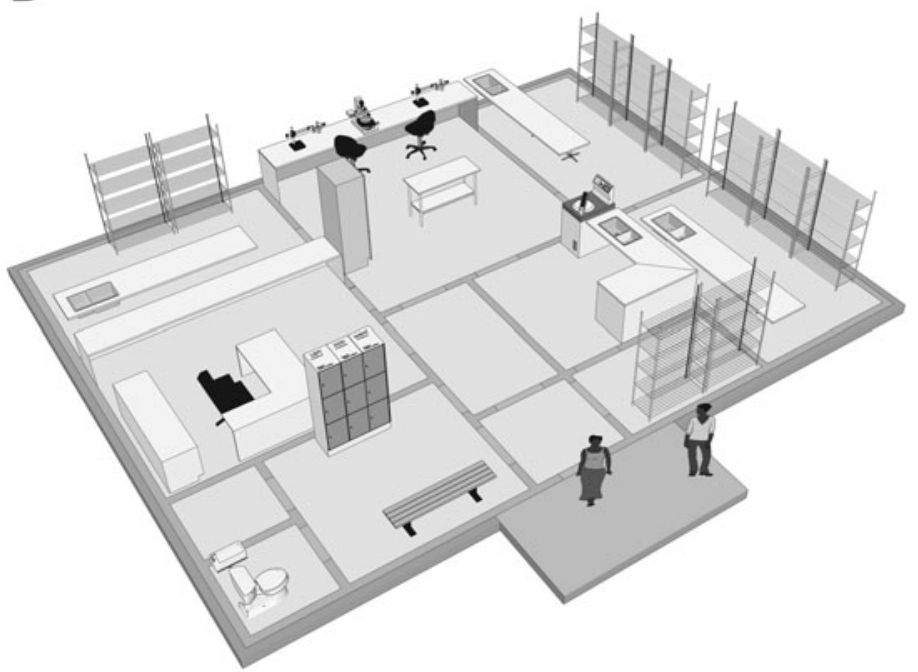

FIG. 2. Generic ACL-2 facility for transgenic mosquito research (A, simple floor plan and $\mathbf{B}$, top view). 
The engagements between architects, engineers, and scientists must be inclusive to ensure that the quality of the material to be used is determined and documented in the design phase. The comprehensive architectural floor plans, together with wall and roof elevations, should be forwarded to the NBA or another authority with the responsibility for approving the design, at the earliest opportunity, and any subsequent changes must be communicated, with implementation of the changes halting until the amendments are approved. In some countries, the NBA conducts the final inspection before official commissioning of the facility for use. In some countries, the regulator may authorize the activities in the facility by issuing permits for specific study activities, rather than approving the facility as a particular level of containment. In addition to requirements for research on genetically modified organisms, established through a biosafety framework, there may be containment regulations that apply to other situations and structures, such as for vector research (Tabachnick 2006), which also must be met.

\section{Renovation of an existing building}

An existing structure can be used as the outer shell to house a containment facility or can be renovated to become a containment facility itself. Use of existing structures can reduce capital input, but only if existing links to electricity, water, and sewage grids allow adaptation for containment measures. Cost savings should not be assumed, as, in fact, new builds might more easily be fitted for containment (Crane and Kreitlein 2006). Renovation of numerous laboratories working with hazardous organisms and substances has been reported for Former Soviet Union sites and in response to the need for facilities during disease outbreaks in Africa (Yeh et al. 2016). There has also been a successful short-term use of BSL-2 laboratories prefabricated in shipping containers and/or installed as modular units (Crane and Kreitlein 2006, Germfree 2016).

Renovation to an ACL-2 facility may be easy to accommodate by adding filters and screens as physical barriers for containment, although design constraints may arise due to the existing configuration of the building and services. Examples of containment facilities employing existing structures include tsetse production units in Slovakia, which provide seed colonies and backup for African facilities (IAEA 2008), although these are not working with transgenic strains.

Compliance for the facility construction or renovation should be checked against all building codes, in addition to laboratory and containment facility requirements. This may vary from location to location and is likely to include municipal as well as national requirements.

\section{Regulated waste}

An important aspect of containment facility design is the incorporation of physical measures to control loss of the study organism through waste disposal. Procedures complement these physical measures to ensure that the fundamental precepts of containment are achieved.

All of the waste leaving a containment facility requires management to prevent any life stage of the research organisms from accidentally being taken out of containment. Solid or liquid waste arising from research of this nature will be regulated in the sense that there are conditions for handling and disposal aimed at maintaining containment of the organism under study. Both transgenic and nontransgenic strains of insects should be treated as "regulated" because of the possibility of cross-contamination of strains, as well as the likelihood that there could be more mistakes if two approaches to cleaning and sorting waste are used in the same facility. All waste is subject to autoclaving, but insects in any life stage should also be killed before being put into the autoclave bags for additional assurance. In the case of research on gene drive, it is essential that the autoclave is accessible inside the designated containment area, not only within the facility (Benedict et al. 2018).

Examples of waste from a transgenic mosquito facility that require special handling (such as being autoclaved before removal from the facility for incineration) are as follows:

- Solid-filter papers used for sieving eggs, toweling used for biological spills, dead mosquitoes of all life stages, leftover diet (sugar and larval diet), consumables, or minor equipment such as air vent filters or sink filters.

- Liquid-culturing water (sieved to remove any life forms) and wash water from trays, small quantities of blood from feeding equipment, and water from hand washing during work in insectaries.

There may also be routine office waste such as paper used by staff, but to avoid confusion, a separate unregulated waste stream is not recommended for research facilities. Obsolete electronic equipment or furnishings must be treated and inspected to ensure that there are no life forms of mosquitoes before their removal. This is also true of laboratory coats taken away for laundering. A separate set of cleaning equipment should be kept in the containment facility, rather than taking equipment in and out of it. None of the above forms of waste, other than the exceptions noted, is classed as hazardous waste under international conventions or those national frameworks that define it.

Care should be taken to review all types of waste generated by the research activities to ensure proper categorization and waste stream handling as an essential aspect of compliance. This includes training of all personnel, including those not involved in research, but entering the facility, such as maintenance or service providers. Documentation of the decisions made in that review should be posted for inspection; training records may be required.

If anything to be used is classed as hazardous, it is worth considering if alternatives exist to avoid having any type of hazardous material in facilities operating in countries where hazardous waste disposal may not meet international standards. Hazardous waste includes waste that is toxic, infectious, or mutagenic, among other properties as described in the European Directive 2008/98/EC on waste (European Commission 2008). Potential sources of hazardous waste are listed in the Basel Convention on the Control of Transboundary Movements of Hazardous Wastes and their Disposal (Basel Convention 1989). For research on uninfected mosquitoes, even the use of animal blood for membrane feeding should not invoke this categorization, owing to the very small quantities being used and proper handling when washing equipment (Ouédraogo et al. 2013). Furthermore, most facilities either purchase blood from a source that tests for pathogens or have testing in-house for both worker safety and the health of the colonies. 
Proper handling of waste, therefore, is essential to ensure containment, but compliance with biomedical or hazardous waste regulations for waste not in those categories is not. If a national or local government framework defines the facility waste as being in such categories, it is worth requesting a review of this classification.

\section{Approval and Authorization}

\section{Determination of the required containment level}

Guidance and regulations on containment facilities often describe specific risk management and biosafety measures in line with increasing levels of containment with increasing risk. Biosafety Levels 1-4 (BSL-1 to BSL-4) are based on a scale of increasing risk from infectious substances (see for example CDC 2009), but BSL-1 is sufficient for transgenic vector species, for example, if they are maintained free of infectious disease and pathogens (WHO 2004, Scott 2005, CDC 2009), and other hazards are not changed by the transformation (e.g., if the vector capacity is the same or less compared with natural populations). * The recommendation for studies on transgenic arthropods is an ACL-2 (ACME/ASTMH 2003), although a gene drive type of modification may require additional considerations (Benedict et al. 2018).

Worker safety or occupational health may require other measures to be carried out (Tabachnick 2006). Procedures should be imposed to avoid introduction of transmissible diseases to the laboratory colonies of vector species. Studies following international guidance on allergenicity testing may be worth pursuing for transgenic biting insects.

\section{Application for certification of the facility and/or import of study organisms}

As with the construction of a building, an application for import of transgenic mosquitoes or permission to conduct studies in containment requires a partner institution that not only leads the process but also commits to responsibility for the outcomes. In Kenya, for example, the Institutional Biosafety Committee, Institutional Ethics Committee, and NBA should each grant approval for an import permit for new organisms. Some countries will certify a facility so that any studies classed at that level should be reported, but do not necessarily need individual approval by the NBA during the period of facility certification. ${ }^{\dagger}$ Other countries, however, will require a separate application for import of a study organism (if developed externally) or for the plan for studies. This allows the NBA to review in more detail the proposed study design, the purpose and possible benefits of the study, and the measures taken outside the facility during transport and delivery. Any changes to the research plan, even in the

*"Biosafety level 1 (BSL-1) is the basic level of protection and is appropriate for agents that are not known to cause disease in normal, healthy humans." This is also used when the research organisms "present minimal potential hazard to laboratory personnel and the environment" (Appendix IV - Laboratory Biosafety Level Criteria; CDC 2009). The WHO (2004) description of biosafety levels does not even use the term "containment" until BSL-3.

'For example, in the UK, new premises designed for lower risk level (Class 1 and 2) must be notified and acknowledged prior to first studies in containment; however, after that, the institution simply notifies about each study to be conducted in the already registered facility. http://www.hse.gov.uk/biosafety/gmo/notifications/what.htm methodologies described in an application, should be carefully considered to determine the need for additional approval or amendments to the permit.

To petition and secure regulatory authorization, the PI for the partner institution submits an application to the appropriate competent national authorities, usually the NBA. Other bodies may have oversight of the proposed research, however, depending on the final target insect populations (agriculture, public health, or environment). Such requirements may not always be anticipated due to the novel type of research being carried out. Engagement with all potential stakeholders in advance of submission may support more streamlined evaluation timelines.

In most instances, a template for applications can be obtained from the NBA. The template may require some adaptation, however, since most were created in the context of genetically modified plants. It is often permissible, in consultation with the NBA, to strike through some sections not relevant to insects and to add other details, where useful to fulfilling the intentions of the regulations (discussed in Quinlan et al. 2018).

In general, the information required will comprise the following categories:

- Information about the responsible parties.

- Information on the organism, in this case, mosquito species and strain.

o Identity of the organism (taxonomy, natural history, some pedigree of the field caught laboratory stock, and details of introgression).

o Phenotypic expression of any modifications (naturally occurring or engineered).

o Context in terms of the conventional target species (nonmodified) for which the import will occur.

- Information about management during shipping and transport.

- Information on the facilities.

o Floor plan and description of physical measures to comply with containment.

o Equipment and procedures for maintaining equipment. o Contingency plan for any breach.

- Information on the technical staff.

o Qualifications, degrees, and certificates.

o Training for the work and hazards related to the studies.

- Information on the studies to be conducted.

The application(s) for import and/or contained studies also details policy safeguards and biosafety procedural commitments, which govern the responsible handling, maintenance, isolation, and containment of the study organisms. The application communicates expectations not only to regulatory authorities but also internally to the partner institute, PI, and researchers who are responsible for the procedures.

It is useful to provide copies of all data and literature cited with the application. This may require obtaining permission from relevant sources (e.g., journals or publishing houses) if it is to be stored electronically and possibly shared further than the NBA. Applications should be prepared and submitted in the official language of the country.

\section{Shipping of genetically modified strains}

When shipping live transgenic insects, the commercial courier company contracted for shipping should provide details on requirements for packaging, labeling and handling 
to satisfy the recipient country authorities. The International Air Transport Association (IATA) is the global organization most often referenced for requirements regarding shipping biological materials. Training and certification in IATA guidance are necessary for most couriers, but information on shipping is available online (e.g. www.un3373.com/un3373packaging/un3245). Researchers must always approach the international courier well ahead of shipping transgenic insects, even for imports into a containment facility. It may be recommended to take charge of a shipment at the airport or other port of entry rather than relying on a local courier to deliver to the research site, when importing transgenic lines. This reduces complications or uncertainty regarding the chain of custody once within the country of destination.

Countries may require additional paperwork or permits to ship species that are non-native to the importing country or vector species under regulations additional to those related to genetic modification. A trial run with the required documentation, but without transgenic insects, may be the best way to establish all requirements. It should be noted that there may be official requirements in terms of permits, documentation, and conditions of transport of live insects, in any life stage, from the perspective of the exporting country as well.

\section{Operational Measures that Complement Facility Design}

The best design of containment facilities is not effective unless matched with operational procedures that complement, reinforce, or verify the same objectives for biosafety.
Some of the procedural or operational steps involved in preparing for containment studies, again using the case of transgenic mosquitoes, are shown in Figure 3, several of which are described more fully in Quinlan et al. (2018). For instance, part of the establishment of basic objectives is to determine if the facility is to be restricted to a single project or multiuse. The former (particularly useful for gene drive, to reduce potential strain contamination) greatly reduces various risks, but the latter may provide an important contribution to the institute's own priorities and research plans. Preparation of procedural documents (standard operating procedures, study protocols, Biosafety Manual, and the related sections in applications) requires dedicated resources of either working groups established across a partnership or individual staff time to draft materials for wider review. General procedures should be put into practice using an existing laboratory strain for some generations before importing transgenic strains, to validate the descriptions and make revisions based on experiences of their use.

Studies performed under a permit require plans for appropriate management and analysis of data (i.e., preparation of data collection templates or technologies). This planning may reveal the need for changes to the studies to achieve statistical validity and answer research questions. After an import or study permit is approved, ongoing monitoring for compliance of the original regulations, the terms laid out in the application, and any additional terms and conditions of approval is imperative to avoid fines or even withdrawal of permits and the research being stopped.

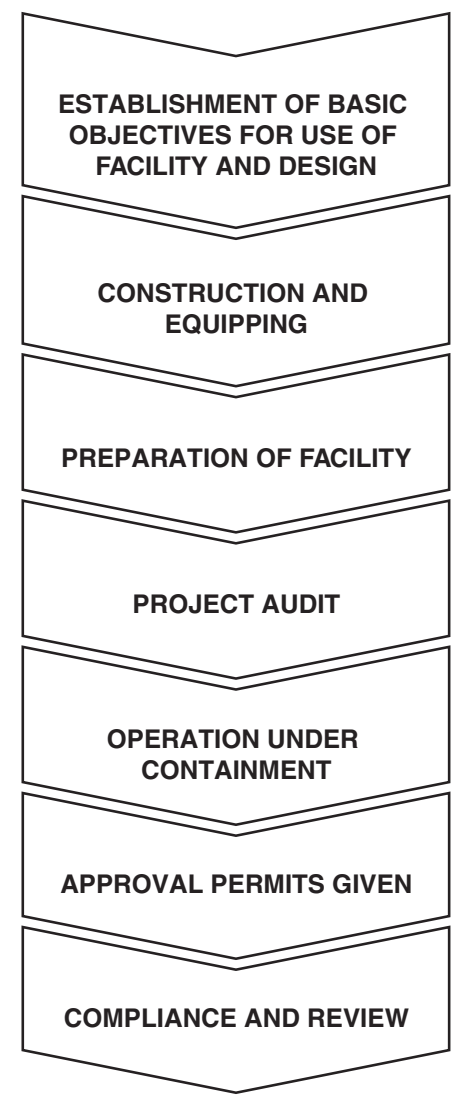

1. Initial risk assessment and determination of ACL-2

2. Understanding of workflow for colony maintenance and studies (consideration of whether same facility will be used to ramp up mosquito numbers for later field studies)

3. Review of all regulatory and legal context, beyond what NBA will address

4. Discussions on need for harmonized environmental conditions or other approaches to colony utility and quality assurance, which may require consideration in design

5. Initial discussions with NBA on anticipated application(s)

6. Safety and access addressed

7. Hiring full complement of staff

8. Equipment procurement, setup, documentation

9. Drafting of Biosafety Manual, SOPs, and other QA documentation

10. Contingency and emergency planning

11. Training in Biosafety Manual and SOPs - document all training

12. Practice shipments of mosquito eggs to test chain of custody, handling, customs processes, and practice handling training strains if available

13. Project audit by institutional colleagues or external experts

14. Completed preparation of databases, templates, and study protocols

15. Submission of regulatory application to NBA

16. Review of application including inspection by NBA

17. Training in protocols or SOPs specific for studies

18. Approval of application and importation permit received

19. Ongoing monitoring of compliance to regulatory requirements, T\&Cs of contained use approval, any ethical approval received (for example, for use of animals for blood meals)

20. Regular review of documentation, i.e. SOPs and training records

ACL, Arthropod Containment Level; NBA, national biosafety authority (generic term); $Q A$, quality assurance; SOP, standard operating procedure; T\&C, terms and conditions.

FIG. 3. Summary of the operational steps performed in preparation of an arthropod containment facility for studies of transgenic mosquitoes in example disease endemic countries. 
For research involving transgenic insects with gene drive, consideration of additional risks and planning will be needed, as discussed in Benedict et al. (2018); some management measures may need to be anticipated and built into the strain, such as unique transgene markers.

\section{Lessons Learned by a Project Importing Transgenic Mosquitoes into Containment in Africa}

Target Malaria is a not-for-profit research consortium that aims to develop and share a novel technology for malaria control, using mass-reared transgenic mosquitoes to reduce mosquito vector populations in countries that have approved the technology's use. ${ }^{\star}$ As part of the progression from a European and North American university-based research program to a broader consortium with African laboratories and field teams, Target Malaria has been preparing containment facilities in African DECs and clarifying the framework for sending transgenic mosquito strains from Europe to West and East African partners. In two cases, ACL-2 facilities were renovated from existing buildings. An existing building was substantially refurbished to the standard of a containment facility at the Institut de Recherche en Sciences de la Santé (IRSS) campus in Bobo Dioulaso, Burkina Faso. The containment facility at the Malaria Research and Training Center (MRTC) at Université des Sciences, des Techniques et des Technologies de Bamako in Mali, has been renovated as an ACL-2 facility from an existing laboratory developed during a previous project (Eggleston and Coulibaly 2010, Coulibaly et al. 2015). These experiences informed the general recommendations offered above.

The project has begun a new-build containment facility in Entebbe, Uganda, on the Uganda Virus Research Institute (UVRI) campus. In this case, a Project Brief similar to an EIA was required under the National Environment Act (NEA) CAP 153 (Uganda 1995) and the EIA Regulations (1998) of Uganda (Uganda 1998). (A full Environmental Impact Study is not conducted unless it is determined that the proposed project/building could have significant impact on the environment.) This Project Brief covered topics on the physical, biological, and sociocultural environment within the project area and was prepared by a trained expert who is certified and registered with by national environmental authorities. According to that report, the facility in question fell under the third schedule to the NEA Act under category 2: Urban Development-establishment. This type of study focuses on the impact during the construction phase, such as dust and debris from excavation, and potential ongoing impacts from waste disposal, water use, noise from operations, increased traffic, and so forth. In this case, the possibility of mosquitoes escaping the facility was noted, but it was considered that all the mitigation measures in place would be adequate to prevent such an occurrence. All the findings of potential impact were considered to be counteracted by the mitigation measures in place or to be used. Despite approval, there is the condition of annual inspections to ensure compliance in the case of Uganda's new mosquito containment facility.

The Project reviewed the issue of waste management, as described above, and determined that none of the waste anticipated from the mosquito research facilities is classed as

\footnotetext{
${ }^{*}$ More information can be found at http://targetmalaria.org
}

hazardous or biomedical. For example, although biomedical waste regulations in Burkina Faso (Burkina Faso 2008) refer to waste originating from "human or veterinary medicine" as biomedical, that decree and the environment code (Burkina Faso 2013) explain that the category includes only waste that is contaminated or could be infectious.

Target Malaria carried out an internal audit of each of the partner facilities. This was done by experts in the various areas of concern, either from within the project or institute, or external, as a key part of the "trial run" approach. This was done before submission of regulatory documentation to identify any remaining gaps in preparations. It was found to promote confidence in the insectary teams, project management, and funders that the correct standards were achieved, before facing official inspections.

Another important training opportunity for Target Malaria was the import and use of a nontransgenic mosquito strain, which required the same handling processes (backcrossing each generation), to maintain a natural color variant mutation arising from the longstanding G3 laboratory strain (an Anopheles gambiae s.1. strain). This variant trait can be visually recognized at a level of precision similar to that of the transgenic marker. This training exercise was run over several months and developed experience and confidence working under such conditions (Sylla et al. 2017).

The Target Malaria Project also used the shipment of these nontransgenic strains, initially from Europe to West Africa, to gain experience in maintaining the chain of custody during shipment and monitoring the transit temperature, humidity, and time expended, and resulting egg quality and hatch rates. The shipping trial runs also provided the opportunity for the private courier company and national customs authorities to experience a similar type of shipment before processing actual transgenic mosquito shipments. Only the eggs of these strains were shipped, to match the anticipated shipment of the transgenic strains. Anopheles gambiae eggs are far more vulnerable to temperature and humidity fluctuations than those of other vector species, particularly Aedes aegypti (e.g., Sota and Mogi 1992). This makes transport of Anopheles eggs a much lower risk in terms of survival in case of an accidental release, but on the other hand reduces the chance of successful shipment for establishment of research colonies. Trial runs are continued until successful import and establishment of nontransgenic strains are achieved, to avoid encountering problems with shipments of transgenic strains.

Mumford et al. (2018) describe some of the data collected by Target Malaria to ensure that containment studies can provide meaningful results for decision making along the stepwise approach described in this article's introduction. In addition to complying with national requirements or following international guidance on containment, the Target Malaria consortium chose to self-impose appropriate good laboratory practices (GLP) (but not try to adapt certified GLP) to achieve several objectives:

- To ensure facility design, equipment, staff organization, and expertise are fit for purpose.

- To instill a culture and awareness of safety, regulations, and compliance.

- To standardize across facilities the most important standard operating procedures and study protocols. 
- To document all processes, methods, and study results in a manner that leaves an auditable data trail, for others to access, if necessary to confirm conclusions.

These experiences confirmed the importance of inclusive planning and detailed advance preparations. The work described preceded the first import into Africa of a transgenic strain of mosquitoes for research purposes (a sterile male strain without persistence), thus demonstrating the success of the Target Malaria research consortium in preparing for containment studies.

\section{Discussion}

Advances in genetic strategies for control of insect vector species often rely on transgenic strains developed outside the countries targeted for their use. The receiving country research partner will face a mix of national regulations and, in their absence, international guidance along with gaps that should be considered in light of specific cases not yet well covered. Preparations of containment facilities, procedures, and capacity to conduct studies of transgenic insects are described, focusing on the example of containment at ACL2 with some comparison to possible requirements for gene drive. Requirements often are unique to studies on transgenic insects, because of the parallel national legislative framework created in most countries to implement the Cartagena Protocol on Biosafety (McLean et al. 2012, Quinlan 2014). Much of the information would relate to any insect species, but the specific experiences described relate to transgenic mosquito facility preparations. The example of research on transgenic vector species requires comparative studies across multiple country sites (Quinlan et al. 2018), and thus some harmonized procedures and quality control (Mumford et al. 2018).

\section{Acknowledgments}

This collaborative work was funded by a grant from the Foundation for the National Institutes of Health (FNIH) through the Vector-Based Control of Transmission: Discovery Research (VCTR) program of the Grand Challenges in Global Health (GCGH) initiative of the Bill \& Melinda Gates Foundation (BMGF) and by the subsequent Target Malaria project. Target Malaria receives core funding from the BMGF and from the Open Philanthropy Project Fund, an advised fund of Silicon Valley Community Foundation. The funder did not limit their ability to develop the approach to facility preparations described. The authors also wish to thank Camilla Beech, Josephine Birungi, John Mumford, and Rebecca Murphy for technical review, and Jana Sikorova for document support.

\section{Author Disclosure Statement}

No competing financial interests exist.

\section{References}

ACME/ASTMH (American Committee of Medical Entomology/ American Society of Tropical Medicine and Hygiene). Arthropod containment guidelines, version 3.1. Vector Borne Zoonotic Dis 2003; 3:61-98.
Balestrino F, Benedict MQ, Gilles JRL. A new larval tray and rack system for improved mosquito mass rearing. J Med Entomol 2012; 49:595-605.

Basel Convention. Basel Convention on the Control of Transboundary Movements of Hazardous Wastes and their Disposal. 1989. Available at www.basel.int/portals/4/basel\% 20convention/docs/text/baselconventiontext-e.pdf

Benedict M, Burt A, Cappuro M, De Barro P, et al. Recommendations for laboratory containment and management of gene drive systems in arthropods. Vector Borne Zoonotic Dis 2018; 18:2-13.

Benedict M, D'Abbs P, Dobson S, Gottlieb M, et al. Guidance for contained trials of vector mosquitoes engineered to contain a gene drive system: Recommendations of a Scientific Working Group. Vector Borne Zoonotic Dis 2008; 8:127166.

Brown DM, Alphey LS, McKemey A, Beech C, et al. Criteria for identifying and evaluating candidate sites for open-field trials of genetically engineered mosquitoes. Vector Borne Zoonotic Dis 2014; 14:291-299.

Burkina Faso. Decret N²008-009/PRES/PM/MS/MCEV du 10 janvier 2008 portant Organisation de la Gestion des Déchets Biomédicaux et Assimilé. 2008. Available at www.vertic.org/ media/National\%20Legislation/Burkina\%20Faso/BF_Decret_ Organisation_Gestion_Dechets_Biomedicaux.pdf

Burkina Faso. Loi N ${ }^{\circ}$ 006-2013/AN portant Code de 1‘Environnement au Burkina Faso. 2013. Available at www.onedd-burkina .info/images/textes_officiels/loi_006_code-environnement.pdf

Burt A. Heritable strategies for controlling insect vectors of disease. Philos Trans R Soc Lond B Biol Sci 2014; 369: 20130432.

CDC (Centers for Disease Control and Prevention). Biosafety in Microbiological and Biomedical Laboratories (BMBL), 5th edn. 2009. Available at www.cdc.gov/biosafety/publications/ bmbl5

Coulibaly MB, Traore SF, Toure YT. Considerations for disrupting malaria transmission in Africa using genetically modified mosquitoes, ecology of Anopheline disease vectors, and current methods of control. In: Adelman ZN, ed. Genetic Control of Malaria and Dengue. Cambridge, MA: Elsevier Science 2015: 55-67.

Crane J, Kreitlein S. Small Biocontainment Facilities: Budget and Schedule Challenges, Part 3. 2006. Available at www. alnmag.com/article/2006/03/small-biocontainment-facilitiesbudget-and-schedule-challenges-part-3

Eggleston P, Coulibaly MB. The Right Way to Tackle Malaria with GM Mosquitoes. SciDevNet, 4 August 2010. Available at: www.scidev.net/global/health/opinion/the-right-way-totackle-malaria-with-gm-mosquitoes.html

European Commission. Directive 2008/98/EC of the European Parliament and of the Council of 19 November 2008 on Waste and Repealing Certain Directives. 2008. Available at http://eur-lex.europa.eu/legal-content/EN/TXT/?uri=celex\% 3A32008L0098

Germfree. BSL-2 Containerized Modular Shipping Container. 2016. Available at www.germfree.com/product-lines/lifescience-labs/bsl-2-labs/bsl-2-containerized-modular

IAEA (International Atomic Energy Authority). Model Business Plan for a Sterile Insect Production Facility. Joint FAO/ IAEA Programme, Nuclear Techniques in Food and Agriculture: Vienna, 2008. Available at www-pub.iaea.org/MTCD/ publications/PDF/IAEA-MBP_web.pdf

Iyaloo DP, Elahee KB, Bheecarry A, Lees RS. Guidelines to site selection for population surveillance and mosquito 
control trials: A case study from Mauritius. Acta Trop 2014; 132 Suppl:S140-S149.

Kenya. The Environmental Management and Co-ordination Act, 1999. CAP. 387. 1999. Available at http://faolex.fao.org/ docs/pdf/ken41653.pdf

Klassen W, Curtis CF. History of the sterile insect technique. In: Dyck VA, Hendrichs J, Robinson AS, eds. The Sterile Insect Technique: Principles and Practice in Area-Wide Integrated Pest Management. Dordrecht: Springer, 2005:3-36.

Lavery JV, Harrington LC, Scott TW. Ethical, social, and cultural considerations for site selection for research with genetically modified mosquitoes. Am J Trop Med Hyg 2008; 79:312-318.

Marris C, Jefferson C. Workshop on "Synthetic Biology: Containment and Release of Engineered Micro-Organisms," 29 April 2013, King's College London: Summary of Discussions. 2013. Available at https://kclpure.kcl.ac.uk/portal/ files/9095328/SB_Containement_and_Release_Workshop_ Summary_of_Discussions_Final.pdf

Marsden CD, Comel A, Lee Y, Sanford MR, et al. An analysis of two island groups as potential sites for trials of transgenic mosquitoes for malaria control. Evol Appl 2013; 6:706-720.

McLean M, Foley ME, Pehu E. The Status and Impact of Biosafety Regulation in Developing Economies since Ratification of the Cartagena Protocol. Joint Departmental Discussion Paper 3. Washington, DC: World Bank, 2012.

Mourya DT, Yadav PD, Majumdar TD, Chauhan DS, et al. Establishment of Biosafety Level-3 (BSL-3) laboratory: Important criteria to consider while designing, constructing, commissioning \& operating the facility in Indian setting. Indian J Med Res 2014; 140:171-183.

Mumford JD, Leach AW, Benedict MQ, Facchinelli L, et al. Maintaining quality of candidate strains of transgenic mosquitoes for studies in containment facilities in disease endemic countries. Vector Borne Zoonotic Dis 2018; 18:31-38.

NASEM (National Academies of Sciences, Engineering, and Medicine). Gene Drives on the Horizon: Advancing Science, Navigating Uncertainty, and Aligning Research with Public Values. Washington, DC: The National Academies Press, 2016.

Ouédraogo AL, Guelbéogo WM, Cohuet A, Morlais I, et al. A protocol for membrane feeding assays to determine the infectiousness of $P$. falciparum naturally infected individuals to Anopheles gambiae. MalariaWorld J 2013; 4:16S.

Quinlan MM. Assessing risk of transgenic insects. In: Benedict, MQ, ed. Transgenic Insects: Techniques and Applications. Wallingford: CABI, 2014:283-305.

Quinlan MM, Birungi J, Coulibaly MB, Diabaté A, et al. Containment studies of transgenic mosquitoes in disease endemic countries: The broad concepts of facilities readiness. Vector Borne Zoonotic Dis 2018; 18:14-20.
Scott TW. Containment of arthropod disease vectors. ILAR J 2005; 46:53-71.

Sota T, Mogi M. Interspecific variation in desiccation survival time of Aedes (Stegomyia) mosquito eggs is correlated with habitat and egg size. Oecologia 1992:90:353-358.

Sylla L, Benedict M, Diallo B, Hoyle S, et al. Preparedness of a Containment Laboratory in Prelude of Genetic Strategies Studies for Mosquito Control in Mali. Poster presented at Malaria Eradication Scientific Alliance Meeting, Kampala, Uganda, 2017.

Tabachnick WJ. Laboratory containment practices for arthropod vectors of human and animal pathogens. Lab Anim (NY) 2006; 35:28-33.

Uganda. National Environment Act (NEA) CAP 153. 1995. Available at www.wipo.int/edocs/lexdocs/laws/en/ug/ug019en .pdf

Uganda. The Environmental Impact Assessment Regulation, S.I. No. 13/1998. 1998. Available at http://nema.go.ug/sites/ all/themes/nema/docs/eia_egulations.pdf

WHO (World Health Organization). Laboratory Biosafety Manual, 3rd edition. Geneva, 2004.

WHO/TDR (World Health Organization/Special Programme for Research and Training in Tropical Diseases). Biosafety for Human Health and the Environment in the Context of the Potential Use of Genetically Modified Mosquitoes (GMMs). Training Manual. 2015. Available at www.who.int/tdr/ publications/biosafety-gmm/en

WHO/TDR and FNIH (World Health Organization/Special Programme for Research and Training in Tropical Diseases and Foundation for the National Institutes of Health). Guidance Framework for Testing Genetically Modified Mosquitoes. 2014. Available at www.who.int/tdr/publications/year/ 2014/guide-fmrk-gm-mosquit/en

Yeh, KB, Adams M, Stamper PD, Dasgupta D, et al. National laboratory planning: Developing sustainable biocontainment laboratories in limited resource areas. Health Secur 2016; 14: 323-330.
Address correspondence to: M. Megan Quinlan Centre for Environmental Policy Imperial College London 1.09 Hamilton Building Silwood Park Campus Ascot, SL5 7PY Berkshire United Kingdom

E-mail: m.quinlan@imperial.ac.uk 